small bones found on the same site have been identified as those of a girl or a small woman. their home or their " workshop" on a sandy knoll only a few feet above the level of the marshes of the Waveney Valley. On this knoll and a neighbouring one there are some saucer-shaped depressions in the ground very suggestive of hut-circles.

W. A. Durr.

Lowestoft.

\section{Graphical Interpolation.}

SiR GEORGE DARWIN has directed attention (Mess. of

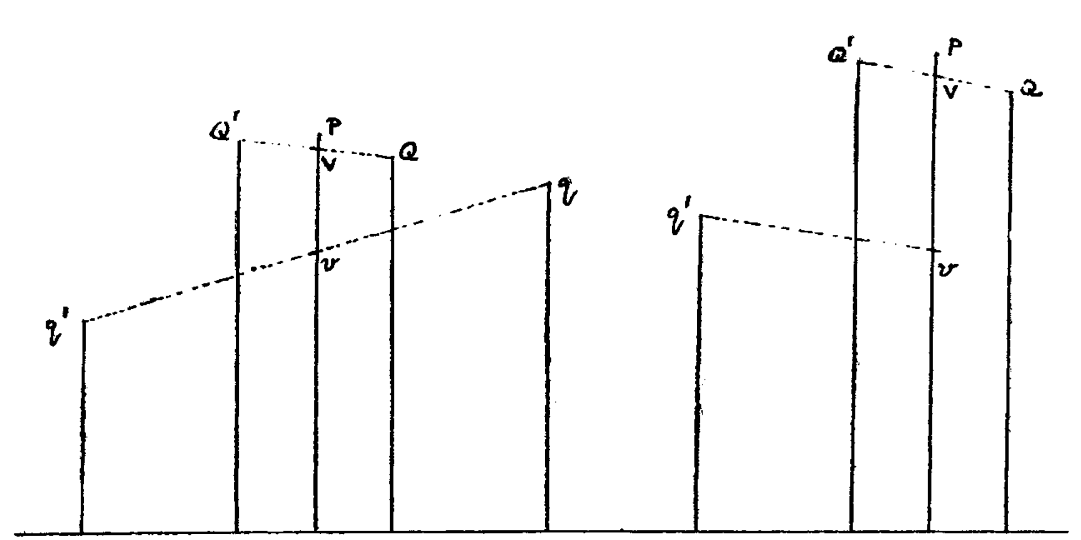

FIG. I.

FIG. 2. the points $Q$.
The makers of the small flint implements evidently had Math., 1877 ; Phil. Trans., A, 1891 ; "Collected Works," vol. i., p. 319 ) to the problem of interpolating values of

as a power series is scarcely justifiable, but it will be seen that it makes it easy to draw a smooth curve through

Merchant Taylors' School, E.C.

\section{Reflection of Polarised Light.}

Some recent correspondence (vol. Ixxvi., p. 637) having directed attention to an error in Preston's "Theory of Light," I venture to send notice of another error in the same work (see article 158 ). The same error will be found in Prof. Tait's article on light (see p. 6Ir, vol. xiv., of the "Encyclopædia Britannica"), and is repeated in his text-book on light (article 271).

1 sent word of the error to the late Sir Greorge Stokes, who expressed himself astonished at it, and said he would look into the matter; but I did not hear from him again, as his letter to me (dated September 19, 1902) was written only five months before his death.

Let the planes of two thin plates of ordinary glass, $\mathrm{A}$ and $\mathrm{B}$, be parallel, so that light, which has been completely plane-polarised by reflection from $A$, falls at the polarising angle upon B. Preston states that this light will be wholly reflected from B, whilst Tait states that this light will be reflected, almost without loss, from $B$.

As a matter of fact, if we represent by unity the intensity of the polarised beam incident upon $\mathrm{B}$, then the intensity of the light reflected

a function at points each half-way between two consecutive points of an equidistant set, e.g. for determining probable half-hourly values when the hourly ones are found from half-hourly values when the hourly ones are found from
observations. Let $q^{\prime}, Q^{\prime}, Q, q$ be four points (Fig. I) the incident beam an infinite number of plates would be with equidistant ordinates $u^{\prime}$, $\mathrm{U}^{\prime}, \mathrm{U}, u$. It is required to find $P$ where the graph through these four points cuts the ordinate half-way between $Q$ and $Q^{\prime}$. By taking the origin on the half-way ordinate and writing the function as

$$
y=a+b x+c x^{2}+d x^{3}+\ldots,
$$

we find that if we neglect terms beyond $x^{3}$, then

$$
a=\frac{U^{\prime}+U}{2}+\frac{\mathrm{I}}{8}\left(\frac{U^{\prime}+U}{2}-\frac{u^{\prime}+u}{2}\right)
$$

A rule for determining the point $P$ is accordingly :-join $\mathbf{Q Q}^{\prime}, q q^{\prime}$ and let them cut the central ordinate in $\mathrm{V}, v$ respectively, then $\mathrm{P}$ lies in $v \mathrm{~V}$ produced, and $\mathrm{PV}=\frac{1}{8} \mathrm{~V} v$. This rule, although theoretically identical, is simpler in form than that discovered by Sir

George Darwin, and seems to be safer, especially near a point of inflexion. It may be worth noticing that in the special case where $Q Q^{\prime}$ and $q q^{\prime}$ are parallel, the cubic reduces to a parabola, and the rule for finding $P$ is involved in the relation $\mathrm{PV}: \mathrm{P} v=\mathrm{QV}^{2}: q v^{2}=\mathrm{I}: 9$. At the beginning and end of the series the rule breaks down, but it can be adapted by assuming the parabolic form for the first and last arcs. In the latter case $q$ is indeterminate, and $q^{\prime} v$ must be drawn parallel to $Q^{\prime} Q$ (Fig. 2).

In the diagram (Fig. 3) the rule is applied to an example in which the assumption that the function can be expressed from $B$ will be represented by about $\frac{2}{7}$, and this takes into account both surfaces of $B$. The remainder of the $v$

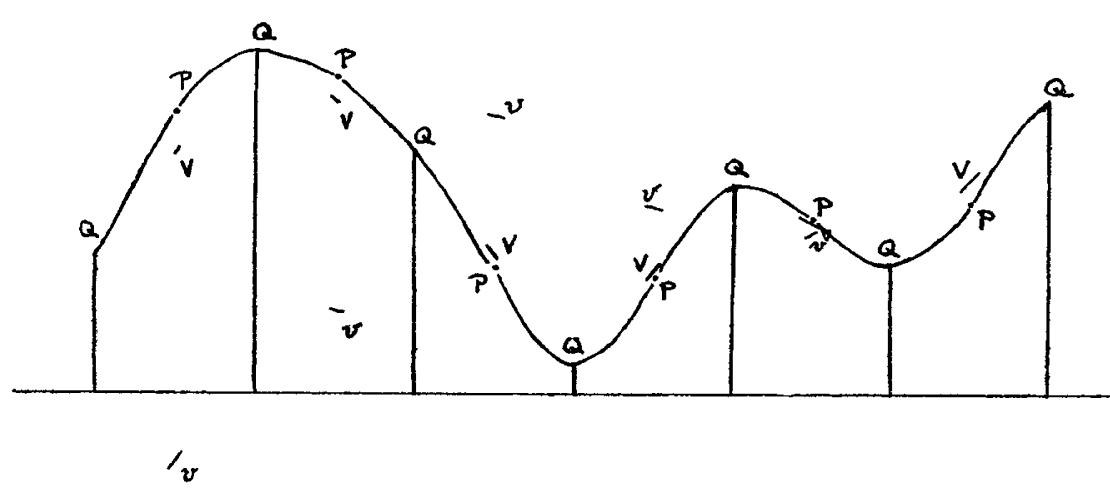

Fig. 3. required, and the glass would have to be perfectly transparent.

Both authors state correctly that, when the plane of reflection of $B$ is perpendicular to that of $A$, and the polarised light from $A$ falls at the polarising angle on $B$, then practically none of this light will be reflected from $B$.

I therefore think that the mistake arose from accidentally supposing that the total want of refiection in the second case should be balanced, as it were, by a complete reflection in the first case.

C. T. WhITMELL.

Invermay, Hyde Park, Leeds. 